\title{
The chemotherapy effect on embryonal rhabdomyosarcoma in a 2-year-old girl in a development country: A rare case
}

\author{
Megasiwi $^{1}$, Widyanti Soewoto ${ }^{2}$, Galih Santoso Putra ${ }^{1}$ \\ From ${ }^{1}$ Resident of Surgery, ${ }^{2}$ Oncology Surgeon, Department of Surgery, Sebelas Maret University, Surakarta, Central Java, Indonesia
}

\begin{abstract}
Rhabdomyosarcoma (RMS) is the third most common solid organ malignancy in children after Wilms tumor and neuroblastoma. It is a highly malignant tumor with local tissue invasion and lymphatic and hematogenous metastases. The main treatment for RMS is complete resection with a combination of chemotherapy and radiotherapy. Here, we present the case of a 2-year-old girl who came to the hospital with a complaint of noisy breathing (Stridor) for the past 2 weeks. Intraoral examination showed an oval mass at the base of the tongue that almost closed the airway, felt soft, and had a bump. A computed tomography scan of the neck was performed, and a solid lesion with clear irregular borders was found in the posterior lingua. Incisional biopsy was performed with histopathological results of embryonal RMS. Two weeks after the biopsy, chemotherapy was performed, and the evaluation after two chemotherapy sessions, the mass size was found to have decreased.
\end{abstract}

Key words: Chemotherapy, Pediatric, Rhabdomyosarcoma

$\mathrm{R}$ habdomyosarcoma (RMS) is a malignant tumor originating from mesenchymal tissue which is suspected to arise from striated muscle cells. RMS is the most common soft-tissue sarcoma in childhood and accounts for 3.5\% of childhood cancers. More than $50 \%$ of tumors occur in the first decade of life [1]. It is a highly malignant tumor with local tissue invasion and lymphatic and hematogenous metastases. Based on the 2013 World Health Organization classification, RMS is divided histologically into four types, namely, embryonal, alveolar, spindle, and pleomorphic. Embryonal RMS and alveolar RMS are the most common types which account for $70-80 \%$ of all cases [2,3]. More than half of the RMS cases are embryonal RMS characterized by the presence of primitive spindle cells, often against a myxoid background $[4,5]$. The main treatment for RMS is complete resection with a combination of chemotherapy and radiotherapy. However, in some cases, especially in difficultto-access areas, complete resection is difficult, thus affecting the prognosis [6].

\section{CASE REPORT}

A 2-year-old girl came with her parents to the Surgical Oncology Polyclinic General Hospital with a complaint of noisy breathing

\section{Access this article online}

Received - 04 September 2021

Initial Review - 19 September 2021

Accepted - 24 September 2021

DOI: $10.32677 /$ ijcr.v7i10.3063
(Stridor). According to her parents, she also began to have difficulty swallowing coarse-textured food and could only swallow softtextured drinks and foods such as porridge. Within 2 weeks, a lump quickly grew on her upper neck. No signs of distant metastases were found. The history of radiation exposure was denied.

Intraoral examination showed an oval mass sized $3 \times 3 \times 4 \mathrm{~cm}$ at the base of the tongue that almost closed the airway, felt soft, and had a bump.

A computed tomography (CT) scan of the neck was performed (Fig. 1) and found a solid mass of $3.5 \times 3.9 \times 5.5 \mathrm{~cm}$ with a firm border and an irregular border on the posterior lingua.

The patient underwent an incisional biopsy. Histopathological results obtained a final diagnosis of embryonal RMS (Fig. 2). The histopathological results were confirmed by immunohistochemical results with positive Myogen obtained according to embryonal RMS. Because the tumor mass was clinically unresectable, 2 weeks after the biopsy, the patient decided to perform neoadjuvant chemotherapy. The neoadjuvant chemotherapy given were vincristine, epirubicin, and etoposide. Evaluation after 2 times chemotherapy showed that the mass size was clinically reduced to $2 \times 2 \times 3 \mathrm{~cm}$.

\section{DISCUSSION}

The most common areas of RMS are in genitourinary (29\%), parameningeal (24\%), extremities $(15 \%)$, retroperitoneal $(13 \%)$,

Correspondence to: Megasiwi, Jl. Kutilang I No 19, Cinderejo Kidul 005/009, Gilingan, Banjarsari, Surakarta. E-mail: drmegasiwi@gmail.com

(C) 2021 Creative Commons Attribution-NonCommercial 4.0 International License (CC BY-NC-ND 4.0). 


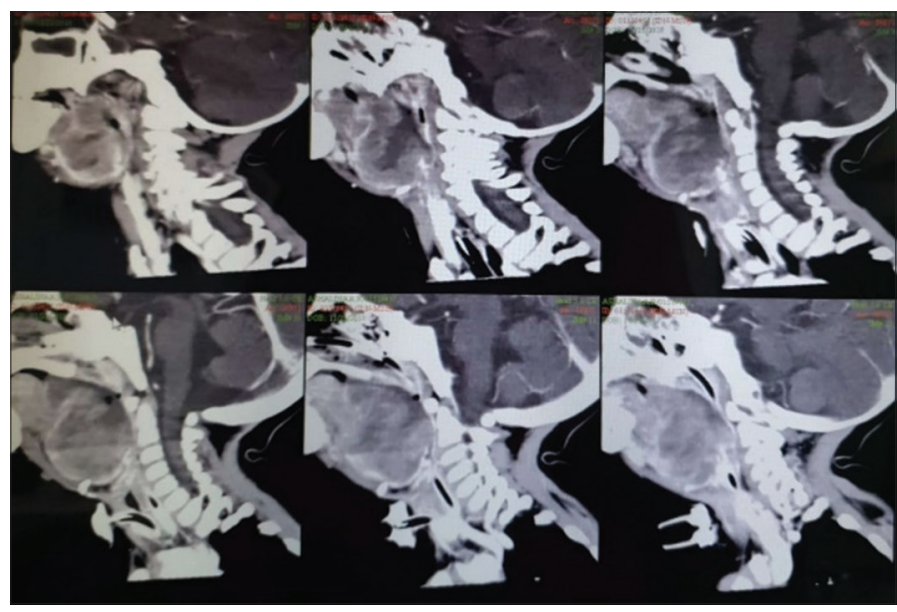

Figure 1: Computed tomography scan of the neck found a solid mass of $3.5 \times 3.9 \times 5.5 \mathrm{~cm}$ with a firm border and an irregular border on the posterior lingua

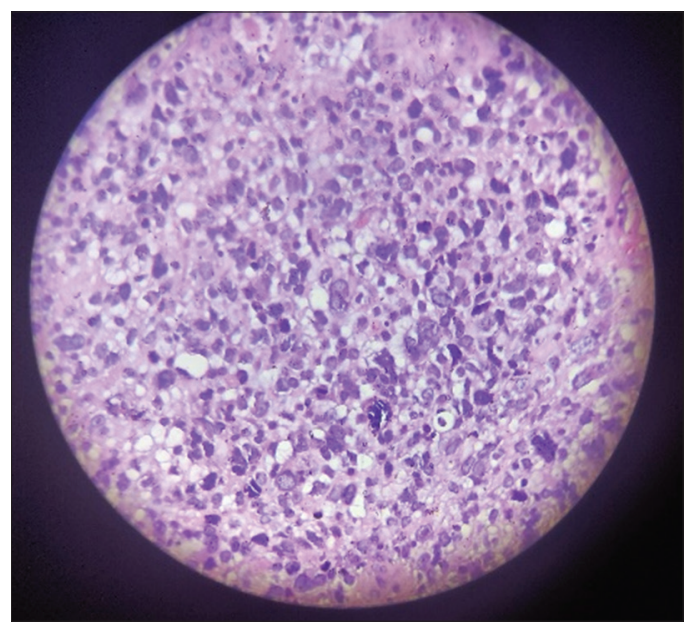

Figure 2: Histology of the tumor showing pleomorphic cells, large nuclei with several multinucleated cells and eosinophilic cytoplasm

orbits $(8 \%)$, head and neck ( $8 \%)$, and other soft-tissue areas (4\%). It often presents as a rapidly growing mass. The manifestations of tumor growth often depend on the location of the primary tumor. For example, tumors of the nasopharynx often cause facial pain or cranial nerve dysfunction, or primary genitourinary tumors may present with intravaginal bleeding and/or symptoms of urinary tract obstruction. Most tumors appear sporadically with no predisposition related to risk factors $[3,4]$.

Our patient was a 2-year-old girl who came with a complaint of noisy breathing. Within 2 weeks, she complained of difficulty swallowing coarse-textured food and a lump on the tongue and neck that grew rapidly. From the data obtained, a mass and obstructions in the airway were found. These data were evidenced by physical examination indicating an oval-shaped, soft and bumpy mass at the base of the tongue that extended posteriorly. The data support the theory that RMS of the tongue and oropharynx most commonly occurs in the first 10 years of age. This tumor has been reported to appear at birth and in the early neonate. Based on the existing data, it is 1.5 times more likely to appear in boys rather than in girls $[2,7,8]$.

The appearance of clinical symptoms found in RMS varies according to the location of the tumor. It usually grows quickly and is painless. For RMS in the head-and-neck area, the clinical manifestation is rapidly growing lesions on the tongue and oropharynx, which can cause symptoms of shortness of breath, dysphagia, cough, and airway obstruction to the point of requiring a tracheostomy. These symptoms are appropriate in this case where a clinical symptom of airway obstruction, namely, stridor, was found so that a tracheostomy was needed $[9,10]$.

The diagnosis was confirmed by a CT scan of the neck and histopathological examination. On CT scan of the neck, there was a solid mass delineated by the irregular border of the posterior lingua. On histological examination, RMS has high histological variability, which describes several levels of skeletal muscle morphology. It is also a highly differentiated neoplasm consisting of rhabdomyoblasts with eosinophilic cytoplasm [4]. Our patient was confirmed by histology followed by immunohistochemistry which obtained positive Myogen. These histopathological results are following the theory that of the five subtypes of RMS, namely, embryonal, alveolar, botryoid, spindle cell, and anaplastic.

Histologically, embryonal RMS is most common and usually consists of elongated pleomorphic tumor cells with a centrally located hyperchromatic nucleus surrounded by a large amount of eosinophilic cytoplasm [11]. This tumor includes primitive mesenchymal cells with various stages of skeletal muscle morphological differentiation, such as strap cells and striated cytoplasm, in loose, myxoid, or cellular collagenous stroma. The diagnosis of solid tumors in most children requires examination of immunohistochemical markers because they often show a nonspecific small round cell tumor phenotype $[4,12]$.

Based on the National Comprehensive Cancer Network (NCCN) guidelines, the management of RMS in children is multidisciplinary. Surgery is the main treatment. If the tumor cannot be resected clinically, chemotherapy and/or neoadjuvant radiotherapy can be performed. In this case, due to the location and size of the inoperable mass, neoadjuvant chemotherapy was performed to reduce the tumor mass [13]. The chemotherapy regimen was given according to the NCCN guidelines, namely, vincristine, epirubicin, and etoposide. In this case, after giving chemotherapy twice, the tumor mass was clinically reduced. Complete resection with histologically free margins is often not feasible without exenteration, but chemotherapy and radiotherapy allow complete remission in most cases. With a combination of therapeutic modalities, namely, chemotherapy, radiotherapy, and surgery, in general, the survival rate for all pediatric RMS s is $71 \%[13,14]$.

\section{CONCLUSION}

A 2-year-old girl was diagnosed with embryonal RMS. The diagnosis was confirmed by the history, physical examination, CT scan, and histopathology. The sacral chordoma is identified by its characteristics. The treatment chosen according to the NCCN guidelines for embryonal RMS is chemotherapy. Vincristine, epirubicin, and etoposide had been performed twice and showed significant results. Chemotherapy can be an initial therapy in cases of unresectable embryonal RMS. 


\section{REFERENCES}

1. Ng WK. Embryonal rhabdomyosarcoma in a young boy. McGill J Med 2007;10:16-9.

2. Bejar DE, Huh WW. Rhabdomyosarcoma in adolescent and young adult patients: Current perspective. Adoles Health Med Ther 2014;5:115-25.

3. Breitfeld P, Meyer WH. Rhabdomyosarcoma: New windows of opportunity. Oncologist 2005;10:518-27.

4. Sebire NJ, Malone M. Myogenin and MyoD1 expression in paediatric rhabdomyosarcomas. J Clin Pathol 2003;56:412-6.

5. NCI. Childhood Rhabdomyosarcoma Treatment; 2013. Available from: http:// www.cancer.gov/cancertopics/pdq/treatment/childrahabdomiosarcoma/ patient. [Last accessed on 2021 Aug 28].

6. Miwa S, Yamamoto N, Hayashi K, Takeuchi A, Igarashi K, Tsuchiya H. Recent advances and challenges in the treatment of rhabdomyosarcoma. Cancers 2020;12:1758.

7. Panda SJ, Chinnaswamy G, Vora T, Prasad M, Bansal D, Kapoor G, et al. Diagnosis and management of rhabdomyosarcoma in children and adolescents: ICMR consensus document. Indian J Pediatr 2017;84:393-402.

8. Dasgupta R, Rodenberg DA. Update on rhabdomyosarcoma. Semin Pediatr Surg 2012;21:68-78.

9. McCarville MB, Spunt SL, Pappo AS. Rhabdomyosarcoma in pediatric patients. Am J Roentgenol 2001;176:1563-9.
10. Pochedly C, Suwansirikul S, Piacitelli J. Rhabdomyosarcoma producing respiratory obstruction. JAMA 1971;217:969.

11. Parham DM, Ellison DA. Rhabdomyosarcomas in adult and children, an update. Arch Pathol Lab Med 2006;130:1454-62.

12. Wachtel M, Runge T, Leuschner I, Stegmaier S, Koscielniak E, Treuner J, et al. Subtype and prognostic classification of rhabdomyosarcoma by immunohistochemistry. J Clin Oncol 2006;24:816-21.

13. National Comprehensive Cancer Network. NCCN Clinical Practice Guidelines in Oncology. Soft Tissue Sarcoma. Version 2020 NCCN. Available from: https:/www.nccn.org/patients/guidelines/content/PDF/ sarcoma-patient.pdf. [Last accessed on 2021 Aug 28].

14. Simon JH, Paulino AD, Rtche JM, Mayr NA, Buatti JM. Presentation, prognostic factors and patterns of failure in adult rhabdomyosarcoma. Sarcoma 2003;7:1-7.

Funding: None; Conflicts of Interest: None Stated.

How to cite this article: Megasiwi, Soewoto W, Putra GS. The chemotherapy effect on embryonal rhabdomyosarcoma in a 2-yearold girl in a development country: A rare case. Indian J Case Reports. 2021;7(10):439-441. 